\title{
The Movember Foundation's GAP3 cohort: a profile of the largest global prostate cancer active surveillance database to date
}

\section{Sophie M. Bruinsma* (D), Liying Zhang ${ }^{\dagger}$, Monique J. Roobol*, Chris H. Bangma*, Ewout W. Steyerberg ${ }^{\ddagger}$, Daan Nieboer** and Mieke Van Hemelrijck ${ }^{\S}(0)$ Movember Foundation's Global Action Plan Prostate Cancer Active Surveillance (GAP3) consortiuma}

\begin{abstract}
*Department of Urology, Erasmus University Medical Centre, Rotterdam, The Netherlands, 'Sunnybrook Health Sciences Centre, University of Toronto, Toronto, ON, Canada, ${ }^{\star}$ Department of Public Health, Erasmus University Medical Centre, Rotterdam, The Netherlands, and ${ }^{\xi}$ Division of Cancer Studies, Translational Oncology and Urology Research, King's College London, London, UK
\end{abstract}

'The Movember Foundation's Global Action Plan Prostate Cancer Active Surveillance (GAP3) consortium members presented in Appendix A.

\section{Objectives}

The Movember Foundation launched the Global Action Plan Prostate Cancer Active Surveillance (GAP3) initiative to create a global consensus on the selection and monitoring of men with low-risk prostate cancer ( $\mathrm{PCa}$ ) on active surveillance (AS). The aim of this study is to present data on inclusion and follow-up for AS in this unique global AS database.

\section{Patients and Methods}

Between 2014 and 2016, the database was created by combining patient data from 25 established AS cohorts worldwide (USA, Canada, Australasia, UK and Europe). Data on a total of 15101 patients were included. Descriptive statistics were used to report patients' clinical and demographic characteristics at the time of PCa diagnosis, clinical follow-up, discontinuation of AS and subsequent treatment. Cumulative incidence curves were used to report discontinuation rates over time.

\section{Results}

At diagnosis, the median (interquartile range $[\mathrm{IQR}]$ ) patient age was $65(60-70)$ years and the median prostate-specific antigen level was $5.4(4.0-7.3) \mathrm{ng} / \mathrm{mL}$. Most patients had clinical stage T1 disease (71.8\%), a biopsy Gleason score of $6(88.8 \%)$ and one tumour-positive biopsy core $(60.3 \%)$. Patients on AS had a median follow-up time of 2.2 (1.05.0) years. After 5, 10 and 15 years of follow-up, respectively, $58 \%, 39 \%$ and $23 \%$ of patients were still on AS. The current version of GAP3 has limited data on magnetic resonance imaging (MRI), quality of life and genomic testing.

\section{Conclusions}

GAP3 is the largest worldwide collaboration integrating patient data from men with PCa on AS. The results will allow individual patients and clinicians to have greater confidence in the personalized decision to either delay or proceed with active treatment. Longer follow-up and the evaluation of MRI, new genomic markers and patient-related outcomes will result in even more valuable data and eventually in better patient outcomes.

\section{Keywords}

adenocarcinoma, guideline, evidence-based, \#PCSM, \#ProstateCancer

\section{Introduction}

Prostate cancer $(\mathrm{PCa})$ is the second most common cancer in men, with nearly a million new cases diagnosed worldwide in 2008[1]. The number of men living with a diagnosis of PCa is likely to continue to increase as the population in many countries continues to age and as cancer is detected earlier, owing to the more widespread use of serum PSA testing $[2,3]$. As a result, active surveillance (AS) was introduced as a management strategy for men with low-risk PCa, with the intention to start curative treatment at the time of progression, and to avoid overtreatment and its associated morbidities. In recent years, AS has evolved from an experimental protocol to a broadly accepted management 
strategy for men diagnosed with low-risk PCa [4]. Contemporary data suggest that use of AS has increased globally [5-7].

Nevertheless, identification of those men whose disease is at low risk for progression is a critical and much debated issue when deciding which men will benefit from AS for their PCa [8]. Numerous agencies have endorsed clinical practice guidelines for the management of low-risk $\mathrm{PCa}$, which include criteria for enrolment of patients in AS programmes and their subsequent management [3]; however, there is currently no consensus in this area. There has been shown to be variability in enrolment criteria and follow-up in international and national series of AS [9]. Moreover, robust data from men with clinically insignificant PCa who are undergoing AS, especially from studies with long follow-up durations, are still limited. Hence, many important questions regarding AS remain unanswered, such as which newly diagnosed men should be considered suitable candidates for AS and what constitutes an appropriate follow-up regimen for AS [10]. There is a need for a worldwide consensus regarding the optimal criteria and protocols for AS and more comparative data on patient selection and testing protocols [11].

In August 2014, the Movember Foundation launched the Global Action Plan Prostate Cancer Active Surveillance initiative (GAP3). Milestones of the project include a global AS database for clinical, marker-related and imaging data. Its primary goal is to create a global consensus on the selection and monitoring of men with low risk PCa. Ultimately, worldwide uniform guidelines will be developed. The aim of the present study was to present data from this unique global dataset on inclusion and follow-up for AS in men with lowrisk PCa.

\section{Patients and Methods}

\section{Study Population}

Between 2014 and 2016, a global database was created by combining patient data from established AS cohorts worldwide. To assemble existing cohorts into a large consortium of cohorts, a new collaborative framework was needed. The GAP3 partners therefore developed documentation required for sharing and use of clinical data within the global database. The database has been developed at the site of Philips Electronics Nederland B.V., Eindhoven, the Netherlands and is currently hosted by the Erasmus Medical Centre, Rotterdam, the Netherlands [12]. The GAP3 initiative was initiated and is coordinated by the Erasmus Medical Centre, Rotterdam, the Netherlands. The Movember Foundation is the sole funder of the project.

Funding has now been secured to provide sustainability of the GAP3 database until February 2019.
Requirements for participation in GAP3 included, among others, ethical approval for sharing digital patient data in a centralized global database, and an active registry of AS patients over the last 2 years or more, including at least $\sim 50$ patients annually. To date, 25 centres from the USA, Canada, Australasia, the UK and Europe fulfilled the requirements for participation and joined the initiative (Table S1). References to the individual AS cohorts can be found in Table S1. The global database currently comprises data on 15101 patients (Table S1; database version 'gap3data_2.3', released in June 2017). A summary of the entry criteria for each individual AS cohort is included in Table S2.

Although many variations in protocols currently exist, most agree that the most suitable patients for AS are those aged $>18$ years, and those with pretreatment clinical stage T1-T2 PCa, serum PSA $\leq 10 \mathrm{ng} / \mathrm{mL}$, a biopsy Gleason score of $\leq 6$ or $(3+4) 7$, and a maximum of two tumour-positive biopsy core samples. Some protocols included PSA density (most often using a threshold of $0.2 \mathrm{ng} / \mathrm{mL}^{2}$ ), the maximum extent of cancer per core (most often using a threshold of 50\%) and life expectancy ( $>10$ years) and adequate biopsy sampling as inclusion criteria for AS. As a result the following baseline host (e.g. age, body mass index, race, ethnicity, marital status, educational level, family history of PCa, smoking history and comorbidities/overall health status) and tumour characteristics (e.g. clinical stage, PSA, prostatic volume, biopsy Gleason score, PSA density, number of biopsy cores with PCa, and maximum cancer extent per core) were recorded.

In addition to baseline information, follow-up information was key for the entire GAP3 project; it will allow us to shed light on current practice and outcomes, with the final goal of providing consensus guidelines. A summary of the monitoring strategy for each individual AS cohort is included in Table S3. After initiation of AS, almost all protocols recommend serial measurement of serum PSA levels, DRE and surveillance biopsy sampling in order to identify pathological progression. Many uncertainties remain surrounding the optimum timing of these surveillance strategies. Some protocols recommend PSA measurements every 3 months, while others state that serum PSA monitoring should be implemented at intervals no more often than every 6 months after the start of AS. Some protocols recommend DRE every 6 months, whilst others do not include DRE in follow-up as a result of the use of MRI. Substantial variation exists in the recommended frequency at which rebiopsy procedures should be conducted. Further, several protocols consider MRI for routine use in AS, again with differences between the recommended frequency, although most protocols recommend a 12-month interval. PSA kinetics and qualityof-life data are less frequently recommended as methods to identify whether or not a patient's cancer has progressed. We therefore collected follow-up information on, for 
example, PSA, PSA kinetics (PSA doubling time and PSA velocity), $T$ stage by DRE, biopsy characteristics and MRI findings (e.g. suspicious lesions found on MRI). Finally, the database contains information on discontinuation of AS (e.g. the reasons for stopping AS), potential subsequent treatments (e.g. radical prostatectomy), and cause of death.

\section{Statistical Analyses}

Descriptive statistics were used to assess the clinical and demographic characteristics at time of PCa diagnosis for all men included in the GAP3 cohort, their clinical follow-up, discontinuation of AS and potential subsequent treatments. Cumulative incidence curves were used to report discontinuation rates over time [13]. $\mathrm{R}$ software was used to perform all analyses [14].

\section{Results}

The GAP3 database currently comprises data on 15101 patients from 25 centres across 15 countries (database version 'gap3data_2.3', released in June 2017). At time of diagnosis, the median (interquartile range [IQR]) patient age was $65(60-70)$ years, the median (IQR) PSA was 5.4 $(4.0-7.3) \mathrm{ng} / \mathrm{mL}$, the median (IQR) PSA density was 0.12 $(0.09-0.17) \mathrm{ng} / \mathrm{mL}$ and the median (IQR) prostate volume was 43.2 (33-59) cc. Most patients had a clinical stage T1 (71.8\%), a biopsy Gleason score of $6(88.8 \%)$, one tumour-positive biopsy core (60.3\%) and no comorbidity (25\%; Table 1). Table S4 shows patient characteristics at time of PCa diagnosis for all patients included in the GAP3 cohort for each participating centre. Patients on AS had a median (IQR) follow-up time (i.e. the time until discontinuation or the time until the last known follow-up without discontinuation being reported) of 2.16 (1.024.47) years. The maximum follow-up time was 21.3 years. The median (IQR) number of years until patients' last follow-up while on AS was $1.99(0.83-4.24)$ years

(Table 2).

By the end of current follow-up, 45 patients $(0.3 \%)$ had developed metastases and 566 patients (3.7\%) had died, of whom $37(0.2 \%)$ died from PCa (Table 2). The main clinical and demographic characteristics and clinical follow-up data for all patients who developed metastases during AS $(n=45)$ and for all patients who developed metastases and died from PCa $(n=17)$ are summarized in Table 3 . Of the patients who had died from PCa by the end of current follow-up $(n=37)$, a total of 32 patients had switched to curative treatment, of whom 21 switched to androgen deprivation therapy, four to external beam radiotherapy, two to external beam radiotherapy and brachytherapy, one to external beam radiotherapy and androgen deprivation, and four to radical prostatectomy.
Table 1 Characteristics at time of prostate cancer diagnosis for all men included in the GAP3 cohort*.

\begin{tabular}{|c|c|c|}
\hline Characteristics & $\begin{array}{l}\text { Distribution of } \\
\text { characteristics } \\
(N=15 \text { 101) }\end{array}$ & $\begin{array}{l}\text { Number of } \\
\text { centres } \\
\text { reported } \\
\left(N_{\text {total }}=25\right)\end{array}$ \\
\hline Median (IQR) age, years & $65(60-70)$ & 25 \\
\hline \multicolumn{3}{|l|}{ Age, $n(\%)$} \\
\hline$\leq 55$ years & $1547(10.3)$ & 25 \\
\hline $56-60$ years & $2402(16.1)$ & \\
\hline $61-65$ years & 3579 (23.9) & \\
\hline $66-70$ years & $4002(26.8)$ & \\
\hline $71-80$ years & $3256(21.8)$ & \\
\hline$>80$ years & $172(1.1)$ & \\
\hline \multicolumn{3}{|l|}{ Year of diagnosis, $n(\%)$} \\
\hline 1992-1997 & $260(1.8)$ & 25 \\
\hline 1998-2004 & 1743 (11.6) & \\
\hline $2005-2008$ & $3011(20.2)$ & \\
\hline 2009-2011 & $4101(27.5)$ & \\
\hline 2012-2014 & 4228 (28.4) & \\
\hline $2015-2016$ & $1565(10.5)$ & \\
\hline \multicolumn{3}{|c|}{ Charlson comorbidity index, $n(\%)$} \\
\hline 0 & $3775(25.0)$ & 10 \\
\hline 1 & $669(4.4)$ & \\
\hline 2 & $761(5.0)$ & \\
\hline$\geq 3$ & $563(3.7)$ & \\
\hline Missing & $9333(61.8)$ & \\
\hline \multicolumn{3}{|l|}{ T stage (at DRE), $n(\%)$} \\
\hline $\mathrm{T} 1$ & $10841(71.8)$ & 23 \\
\hline T2 & $2034(13.5)$ & \\
\hline $\mathrm{T} 3$ & $11(0.1)$ & \\
\hline $\mathrm{T} 4$ & $1(<0.1)$ & \\
\hline Unknown & $2214(14.6)$ & \\
\hline \multicolumn{3}{|l|}{ Gleason grade group, $n(\%)$} \\
\hline$<6$ & $400(2.7)$ & 25 \\
\hline 6 & $13198(88.8)$ & \\
\hline$>6$ & $1263(8.5)$ & \\
\hline Unknown & $240(1.6)$ & \\
\hline \multicolumn{3}{|l|}{ PSA level, $n(\%)$} \\
\hline $0-3.0 \mathrm{ng} / \mathrm{mL}$ & $1826(12.6)$ & 25 \\
\hline $3.1-6.0 \mathrm{ng} / \mathrm{mL}$ & $6913(47.8)$ & \\
\hline $6.1-10.0 \mathrm{ng} / \mathrm{mL}$ & $4511(31.2)$ & \\
\hline$>10.0 \mathrm{ng} / \mathrm{mL}$ & $1207(8.3)$ & \\
\hline Median (IQR) PSA, ng/mL & $5.4(4.0-7.3)$ & \\
\hline Missing PSA data, $n(\%)$ & $644(4.3)$ & \\
\hline \multicolumn{3}{|l|}{ Prostate volume } \\
\hline Median (IQR), cc & $43.2(33.0-59.0)$ & 22 \\
\hline Missing, $n(\%)$ & $4069(26.9)$ & \\
\hline \multicolumn{3}{|l|}{ PSA density } \\
\hline $\begin{array}{l}\text { Median (IQR), } \\
\text { ng/mL }\end{array}$ & $0.12(0.09-0.17)$ & 22 \\
\hline Missing, $n(\%)$ & $4221(28.0)$ & \\
\hline \multicolumn{3}{|l|}{ Positive cores } \\
\hline Median (IQR) & $1(1-2)$ & 24 \\
\hline Missing, $n(\%)$ & $1305(8.6)$ & \\
\hline \multicolumn{3}{|l|}{ Positive cores } \\
\hline 0 & $78(0.6)$ & 24 \\
\hline 1 & $8321(60.3)$ & \\
\hline 2 & $3270(23.7)$ & \\
\hline$\geq 3$ & $2127(15.4)$ & \\
\hline \multicolumn{3}{|c|}{ Percentage of cancer in any one core } \\
\hline Median (IQR) & $10(5-20)$ & 17 \\
\hline Minimum, maximum, $\%$ & 0,100 & \\
\hline Missing, $n(\%)$ & $6114(40.5)$ & \\
\hline
\end{tabular}

$I Q R$, interquartile range. *Database version 'gap3data_2.3', released in June 2017. 
음

하은

$\geq$

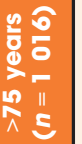

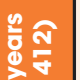

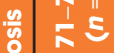

응 สิ

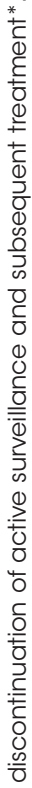

$\frac{0}{20}$

$\stackrel{0}{+0}$

$\frac{2}{0}$

$\frac{\overline{0}}{\overline{\frac{D}{0}}}$

N

$\frac{0}{\circ}$

过

क्ष

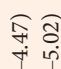

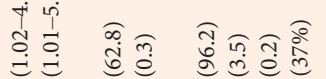

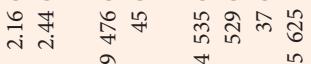

$\pm$

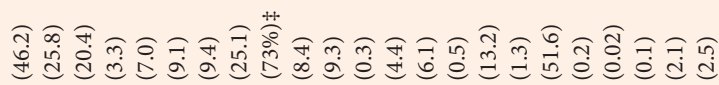

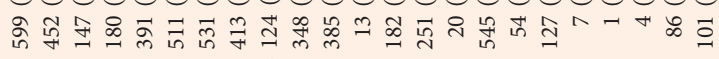<smiles>[C-]1=CCCC1</smiles>

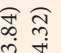

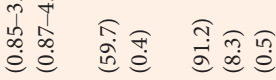

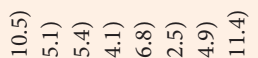

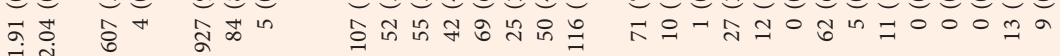

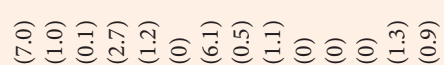

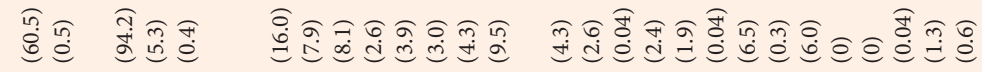

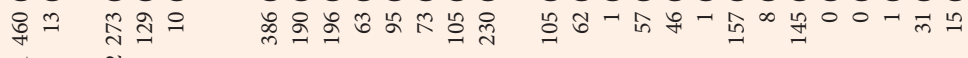

ลิำ

站

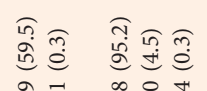

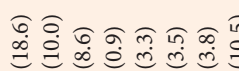

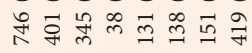

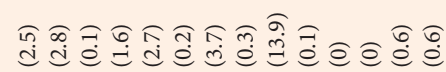

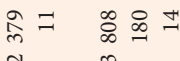

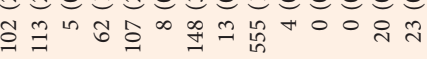

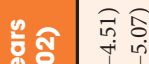

䙳

तु

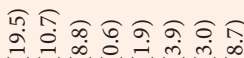

สุล

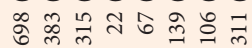

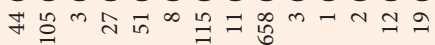

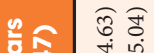

tis

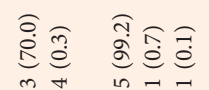

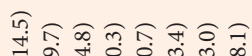

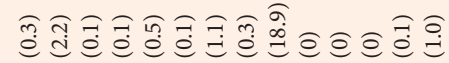

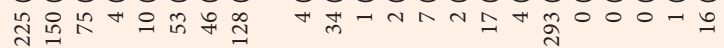

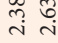

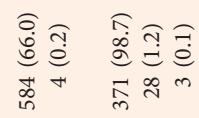

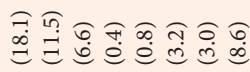

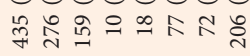

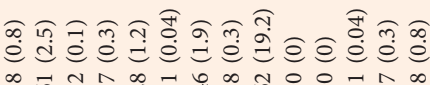

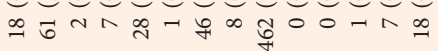
学蓄

这苛

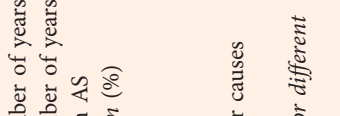

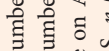

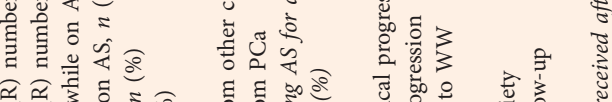

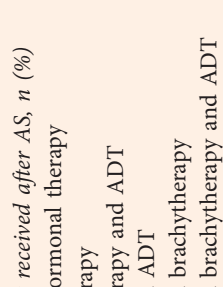

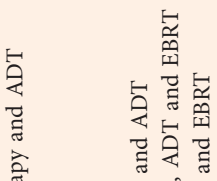

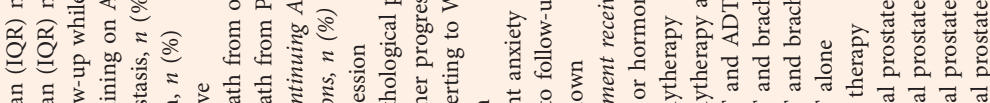

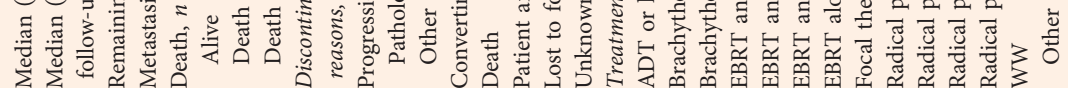

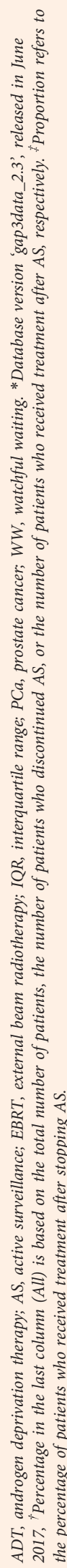


Table 3 Clinical and demographic characteristics and clinical follow-up for all patients who developed metastases during active surveillance and for all patients who developed metastases and died from prostate cancer*

\begin{tabular}{|c|c|c|}
\hline Characteristics & $\begin{array}{c}\text { Distribution of } \\
\text { characteristics } \\
\text { of patients who } \\
\text { developed } \\
\text { metastases } \\
(N=45)\end{array}$ & $\begin{array}{l}\text { Distribution of } \\
\text { characteristics of } \\
\text { patients who } \\
\text { developed } \\
\text { metastases } \\
\text { and died from } \\
\text { PCa }(N=17)\end{array}$ \\
\hline Median (IQR) age, years & $66(62-72)$ & $66(64-72)$ \\
\hline \multicolumn{3}{|c|}{ Charlson comorbidity index, $n(\%)$} \\
\hline 0 & $45(100)$ & $17(100)$ \\
\hline 1 & $0(0)$ & $0(0)$ \\
\hline 2 & $0(0)$ & $0(0)$ \\
\hline$\geq 3$ & $0(0)$ & $0(0)$ \\
\hline Missing & $0(0)$ & $0(0)$ \\
\hline \multicolumn{3}{|l|}{$\mathrm{T}$ stage (at DRE), $n$ (\%) } \\
\hline $\mathrm{T} 1$ & $21(46.7)$ & $6(35.3)$ \\
\hline $\mathrm{T} 2$ & $15(33.3)$ & $7(41.2)$ \\
\hline $\mathrm{T} 3$ & $2(4.4)$ & $1(5.9)$ \\
\hline $\mathrm{T} 4$ & $1(2.2)$ & $1(5.9)$ \\
\hline Unknown & $6(13.3)$ & $2(11.8)$ \\
\hline \multicolumn{3}{|c|}{ Gleason grade group, $n$ (\%) } \\
\hline$<6$ & $3(6.7)$ & $0(0)$ \\
\hline 6 & $28(62.2)$ & $10(58.8)$ \\
\hline$>6$ & $21(46.7)$ & $5(29.4)$ \\
\hline Unknown & $4(8.9)$ & $2(11.8)$ \\
\hline \multicolumn{3}{|l|}{ PSA ng/mL, $n(\%)$} \\
\hline Median (IQR) & $6.9(4.8-8.7)$ & $7.9(4.3-12.5)$ \\
\hline Missing, $n(\%)$ & $4(8.9)$ & $1(5.9)$ \\
\hline \multicolumn{3}{|l|}{ Prostate volume, $\mathrm{cc}$} \\
\hline Median (IQR) & $44(31-55)$ & $41(29-50)$ \\
\hline Missing, $n(\%)$ & $23(51.1)$ & $9(52.9)$ \\
\hline \multicolumn{3}{|l|}{ PSA density $\mathrm{ng} / \mathrm{mL} / \mathrm{mL}$} \\
\hline Median (IQR) & $0.14(0.10-0.19)$ & $0.14(0.11-0.19)$ \\
\hline Missing, $n$ (\%) & $25(55.6)$ & $10(58.8)$ \\
\hline \multicolumn{3}{|l|}{ Positive cores, $n$ (\%) } \\
\hline 0 & - & - \\
\hline 1 & $14(42.4)$ & $5(29.4)$ \\
\hline 2 & $10(30.3)$ & $4(23.5)$ \\
\hline$\geq 3$ & $9(27.3)$ & $3(17.6)$ \\
\hline $\begin{array}{l}\text { Median (IQR) time to } \\
\text { metastasis, years }\end{array}$ & $6.4(3.5-9.9)$ & - \\
\hline $\begin{array}{l}\text { Median (IQR) time } \\
\text { to death, years }\end{array}$ & - & $10.0(6.1-12.7)$ \\
\hline
\end{tabular}

$I Q R$, interquartile range; $P C a$, prostate cancer. *Database version 'gap3data_2.3', released in June 2017.

A total of 5625 patients (37\%) discontinued AS for the following reasons: $46.2 \%$ for protocol-based progression; $3.3 \%$ switched to watchful waiting; $9.1 \%$ discontinued through patient or clinician choice; $7.0 \%$ died; and $25.1 \%$ discontinued for unknown reasons. For all patients who discontinued AS, treatment was reported in $73 \%$ of the cases $(n=4124)$. Treatment after discontinuation was radical prostatectomy in $51.6 \%$ of patients, external beam radiotherapy in $13.2 \%$ of patients, brachytherapy in $9.3 \%$ of patients and primary androgen deprivation/hormonal therapy in $8.4 \%$ of patients (Table 2). Figure 1 shows the cumulative incidence curves of reasons for discontinuing AS. The percentage of total area shaded for each colour in the figure can be interpreted, at any
Fig. 1 Discontinuation of active surveillance (AS) over time ( $n=14033$ ). Protocol-based progression indicates clinical and pathological progression, clinical progression, other PSA kinetics, pathological progression, PSA progression (PSA doubling time $<3$ years), or radiological progression.

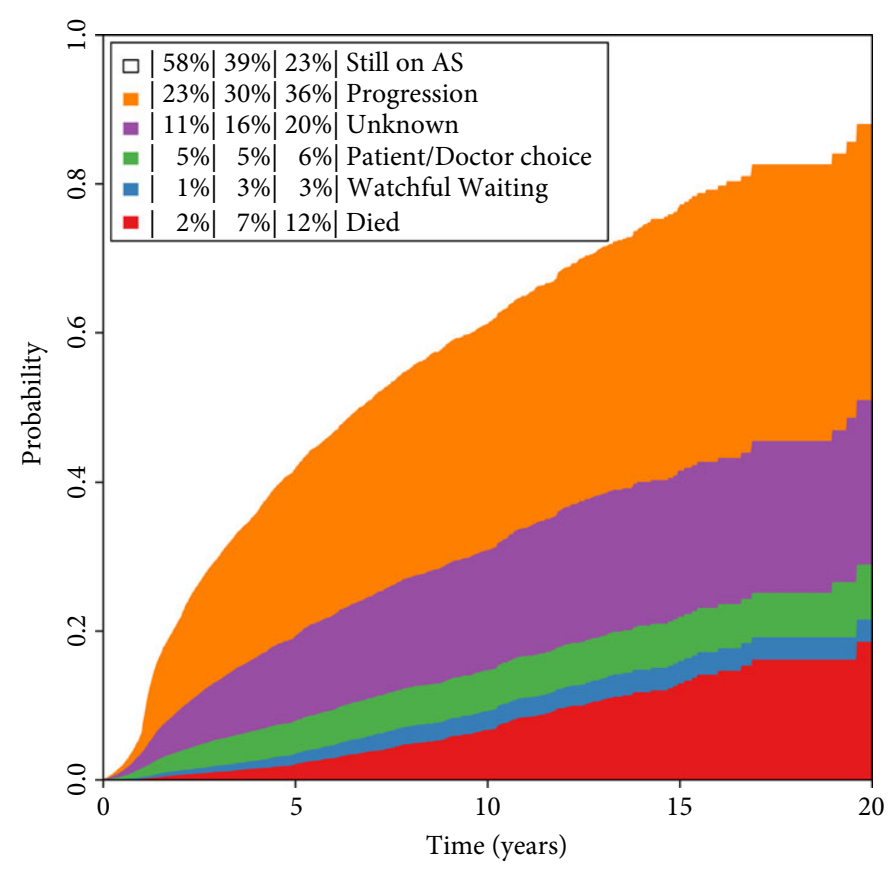

time point, as the risk of discontinuing AS for that stated reason.

Of the 15101 patients, 1068 patients (7.1\%) did not yet have available follow-up data. Among the remaining 14033 patients, after 5, 10 and 15 years of follow-up, respectively, $58 \%, 39 \%$ and $23 \%$ of patients were still on AS, $23 \%, 30 \%$ and $36 \%$ discontinued because of protocol-based progression, $5 \%, 5 \%$ and $6 \%$ discontinued through patient or clinician choice, $1 \%, 3 \%$ and $3 \%$ switched to watchful waiting, $2 \%, 7 \%$ and $12 \%$ died (mostly from another cause), and 11\%, 16\% and $20 \%$ discontinued for unknown reasons.

\section{Discussion}

In recent years, AS has evolved from an experimental protocol to become a broadly accepted, in fact, preferred, management strategy for men diagnosed with low-risk PCa [15]. Nevertheless, consensus on inclusion criteria, surveillance schedules and intervention thresholds for AS of men with low-risk PCa is currently lacking. With this in mind, the Movember Foundation launched the GAP3 initiative.

Several findings deserve particular attention. GAP3 is the largest effort of its type to integrate patient data from men with PCa on AS. With $>15000$ patients, the Movember AS database is the largest centralized PCa AS database to date, 
comprising the majority of the world's AS patient data. Large volumes of AS data have been collected routinely for many years by the affiliated centres worldwide; hence, the central data source enables comparisons of determinants for inclusion and follow-up in AS, and subsequent clinical outcomes (e.g. disease progression), between cohorts and countries and it allows us to determine variable patterns over time. Data capture is nearly complete (i.e. available for at least $90 \%$ of the centres) for key variables such as: serum PSA levels, Gleason score and clinical stage at time of PCa diagnosis; serum PSA levels, T stage by DRE and biopsy characteristics during follow-up; and reasons for discontinuing AS, treatment choices and cause of death. The database thus has a significant amount of highly informative patient data on AS for low-risk PCa. It can therefore make significant contributions to the development of evidencebased consensus guidelines for AS, and as a result, improve the lives of men diagnosed with low-risk PCa.

There are some limitations that need to be considered when using data from GAP3. The database is 'ambidirectional', meaning that it has both a retrospective and a prospective component. Up until now, the GAP3 database has been purely a retrospective database. As a consequence, there was limited control over data collection, and the data of interest were sometimes incomplete or inconsistently measured. For instance, in 18 cohorts the reason for discontinuation of AS is not available. For future analyses, the individual centres will be requested to supply the missing data (if available). During the course of the GAP3 project, it has become apparent that there is an urgent need to assess the value of MRI with respect to disease monitoring in patients on AS. The current patient series has only limited imaging data from MRI, and currently, almost no data are available for quality of life and genomic testing. However, additional funding has now been secured from the Movember Foundation to sustain the database and to add a prospective element, thereby providing the opportunity to collect evidence on imaging (MRI), molecular (genomics) markers, patient-related outcomes and more.

Metastatic disease or death from PCa are ultimate endpoints by which AS should be evaluated [16]; however, because of the slow-growing nature of low-risk PCa, prospective evaluation of these endpoints requires at least another 10-15 years of follow-up [16]. To date, mainly data from non-mature prospective clinical trials of AS, which have a mean follow-up of $<10$ years, are available. The GAP3 database currently also has limited follow-up time, but will in future provide the main resource of real-world data on AS management.

In the global database, PCa death and metastasis remain rare events (both $<1 \%$ ). Current analyses therefore make use of surrogate endpoints such as discontinuation of AS and/or changes in PCa treatment. Nevertheless, follow-up is ongoing until at least 2019, so that in the future GAP3 will contain even more valuable data and provide better insight into patient outcomes.

Active surveillance is evolving into a well-accepted management strategy for appropriately selected men. Unless the over-diagnosis of indolent $\mathrm{PCa}$ is reduced by alternative diagnostic strategies, AS will continue to play an important role. The GAP3 initiative will make significant contributions to this field of research by offering standard, evidence-based guidelines [3]. Clinicians will be able to use these guidelines more confidently to identify patients who are suitable for AS and also to decide whose PCa has progressed and will, therefore, require treatment. Such guidelines will provide reassurance to patients that they have made the best treatment choice for their type of disease [3]. Longer followup, achieved by ongoing commitment of GAP3 participating centres, and the evaluation of, for instance, imaging and new biomarkers, will result in more valuable data and eventually in better patient outcomes.

\section{Acknowledgements}

This work was supported by the Movember Foundation. The funder did not play any role in the study design, collection, analysis or interpretation of data, or in the drafting of this paper. For information, contact Dr. M.J. Roobol: m.roobol@erasmusmc.nl.

\section{Conflict of Interest}

None declared.

\section{References}

1 Center MM, Jemal A, Lortet-Tieulent J et al. International variation in prostate cancer incidence and mortality rates. Eur Urol 2012; 61: 107992

2 Kim TH, Jeon HG, Choo SH et al. Pathological upgrading and upstaging of patients eligible for active surveillance according to currently used protocols. Int J Urol 2013; 21: 377-81

3 Bruinsma SM, Bangma CH, Carroll PR et al. Active surveillance for prostate cancer: a narrative review of clinical guidelines. Nat Rev Urol 2016; 13: 151-67

4 Ganz PA, Barry JM, Burke W et al. National Institutes of Health Stateof-the-Science Conference: role of active surveillance in the management of men with localized prostate cancer. Ann Intern Med 2012; 156: 591-5

5 Tosoian JJ, Carter HB, Lepor A, Loeb S. Active surveillance for prostate cancer: current evidence and contemporary state of practice. Nat Rev Urol 2016; 13: 205-15

6 Loeb S, Berglund A, Stattin P. Population based study of use and determinants of active surveillance and watchful waiting for low and intermediate risk prostate cancer. J Urol 2013; 190: 1742-9

7 Weerakoon M, Papa N, Lawrentschuk $\mathrm{N}$ et al. The current use of active surveillance in an Australian cohort of men: a pattern of care analysis from the Victorian Prostate Cancer Registry. BJU Int 2015; 115(Suppl 5): $50-6$

8 Klotz L. Active surveillance for men with early prostate cancer. 2016. 
9 Philippou Y, Raja H, Gnanapragasam VJ. Active surveillance of prostate cancer: a questionnaire survey of urologists, clinical oncologists and urology nurse specialists across three cancer networks in the United Kingdom. BMC Urol 2015; 15: 52

10 Hall IJ, Richardson LC. Commentary on the State-of-the-Science Conference on the role of active surveillance in the management of men with localized prostate cancer. J Natl Cancer Inst Monogr 2012;2012:135-9

11 Loeb S. Re: Long-term follow-up of a large active surveillance cohort of patients with prostate cancer. Eur Urol 2015; 68: 907

12 Hulsen $\mathrm{T}$, Obbink $\mathrm{H}$, van der Linden $\mathrm{W}$ et al. Integrating large datasets for the Movember Global Action Plan on active surveillance for low risk prostate cancer. Eur Urol Suppl 2016; 15: e958

13 Fine JP, Gray RJ. A proportional hazards model for the subdistribution of a competing risk. J Am Stat Assoc 1999; 94: 496-509

14 RStudio Team. RStudio: Integrated Development for R. Boston, MA: RStudio, Inc., 2015 Available at: http://www.rstudio.com/

15 Cooperberg MR. Long-term active surveillance for prostate cancer: answers and questions. J Clin Oncol 2015; 33: 238-40

16 Welty CJ, Cooperberg MR, Carroll PR. Meaningful end points and outcomes in men on active surveillance for early-stage prostate cancer. Curr Opin Urol 2014; 24: 288-92

\section{Appendix A}

\section{Members of the Movember Foundation's Global Action Plan Prostate Cancer Active Surveillance (GAP3) consortium}

Principle Investigators: Bruce Trock (Johns Hopkins University, The James Buchanan Brady Urological Institute, Baltimore, USA), Behfar Ehdaie (Memorial Sloan Kettering Cancer Center, New York, USA), Peter Carroll (University of California San Francisco, San Francisco, USA), Christopher Filson (Emory University School of Medicine, Winship Cancer Institute, Atlanta, USA), Jeri Kim (MD Anderson Cancer Centre, Houston, USA), Todd Morgan (University of Michigan and Michigan Urological Surgery Improvement Collaborative, Michigan, USA), Laurence Klotz (University of Toronto, Sunnybrook Health Sciences Centre, Toronto, Ontario, Canada), Tom Pickles (University of British Columbia, BC Cancer Agency, Vancouver, Canada), Eric Hyndman (University of Calgary, Southern Alberta Institute of Urology, Calgary, Canada), Caroline M. Moore (University College London \& University College London Hospital Trust, London, UK), Vincent Gnanapragasam (University of Cambridge \& Cambridge University Hospitals NHS Foundation Trust, Cambridge, UK), Mieke Van Hemelrijck (King's College London, London, UK \& Guy's and St Thomas' NHS Foundation Trust, London, UK), Prokar Dasgupta (Guy's and St Thomas' NHS Foundation Trust, London, UK), Chris Bangma (Erasmus Medical Center, Rotterdam, The Netherlands), Monique Roobol (Erasmus Medical Center, Rotterdam, The Netherlands), Arnauld Villers (Lille University Hospital Center, Lille, France), Antti Rannikko (Helsinki University and Helsinki University Hospital, Helsinki, Finland), Riccardo Valdagni (Department of Oncology and Hemato-oncology, Università degli Studi di
Milano, Radiation Oncology 1 and Prostate Cancer Program, Fondazione IRCCS Istituto Nazionale dei Tumori, Milan, Italy), Antoinette Perry (University College Dublin, Dublin, Ireland), Jonas Hugosson (Sahlgrenska University Hospital, Göteborg, Sweden), Jose Rubio-Briones (Instituto Valenciano de Oncología, Valencia, Spain), Anders Bjartell (Skåne University Hospital, Malmö, Sweden), Lukas Hefermehl (Kantonsspital Baden, Baden, Switzerland), Lee Lui Shiong (Singapore General Hospital, Singapore, Singapore), Mark Frydenberg (Monash Health; Monash University, Melbourne, Australia), Yoshiyuki Kakehi (Kagawa University Faculty of Medicine, Kagawa, Japan), Byung Ha Chung (Gangnam Severance Hospital, Yonsei University Health System, Seoul, Republic of Korea).

Pathologist: Theo van der Kwast (Princess Margaret Cancer Centre, Toronto, Canada).

Technology Research Partners: Henk Obbink (Royal Philips, Eindhoven, the Netherlands), Wim van der Linden (Royal Philips, Eindhoven, the Netherlands), Tim Hulsen (Royal Philips, Eindhoven, the Netherlands), Cees de Jonge (Royal Philips, Eindhoven, the Netherlands).

Regional statisticians: Mike Kattan (Cleveland Clinic, Cleveland, Ohio, USA), Ji Xinge (Cleveland Clinic, Cleveland, Ohio, USA), Kenneth Muir (University of Manchester, Manchester, UK), Artitaya Lophatananon (University of Manchester, Manchester, UK), Michael Fahey (Epworth HealthCare, Melbourne, Australia), Ewout Steyerberg (Erasmus Medical Center, Rotterdam, The Netherlands), Daan Nieboer (Erasmus Medical Center, Rotterdam, The Netherlands); Liying Zhang (University of Toronto, Sunnybrook Health Sciences Centre, Toronto, Ontario, Canada).

Clinical Research Partners' IT Experts: Wei Guo (Johns Hopkins University, The James Buchanan Brady Urological Institute, Baltimore, USA), Tanya Milan (Memorial Sloan Kettering Cancer Center, New York, USA), Nicole Benfante (Memorial Sloan Kettering Cancer Center, New York, USA), Janet Cowan (University of California San Francisco, San Francisco, USA), Dattatraya Patil (Emory University School of Medicine, Winship Cancer Institute, Atlanta, USA), Rachel Sanford (MD Anderson Cancer Centre, Houston, Texas, USA), Tae-Kyung Kim (University of Michigan and Michigan Urological Surgery Improvement Collaborative, Ann Arbor, Michigan, USA), Alexandre Mamedov (University of Toronto, Sunnybrook Health Sciences Centre, Toronto, Ontario, Canada), Vincent LaPointe (University of British Columbia, BC Cancer Agency, Vancouver, Canada), Trafford Crump (University of Calgary, Southern Alberta Institute of Urology, Calgary, Canada), Rifat Hamoudi (University College London \& University College London Hospital Trust, London, UK), Jenna Kimberly-Duffell (University of Cambridge \& Cambridge University Hospitals NHS Foundation Trust, 
Cambridge, UK), Aida Santaolalla (King's College London, London, UK \& Guy's and St Thomas' NHS Foundation Trust, London, UK), Daan Nieboer (Erasmus Medical Center, Rotterdam, The Netherlands), Jonathan Olivier (Lille University Hospital Center, Lille, France), Emanuele Bianchi Janetti (Fondazione IRCCS Istituto Nazionale dei Tumori di Milano, Milan, Italy), Tiziana Rancati (Fondazione IRCCS Istituto Nazionale dei Tumori di Milano, Milan, Italy), Helén Ahlgren (Sahlgrenska University Hospital, Göteborg, Sweden), Juanma Mascarós (Instituto Valenciano de Oncología, Valencia, Spain), Annica Löfgren (Skåne University Hospital, Malmö, Sweden), Kurt Lehmann (Kantonsspital Baden, Baden, Switzerland), Catherine Han Lin (Monash University and Epworth HealthCare, Melbourne, Australia), Hiromi Hirama (Kagawa University, Kagawa, Japan), Kwang Suk Lee (Gangnam Severance Hospital, Yonsei University Health System, Seoul, Republic of Korea).

Research Advisory Committee: Guido Jenster (Erasmus MC, Rotterdam, The Netherlands), Anssi Auvinen (University of Tampere, Tampere, Finland), Anders Bjartell (Skåne University Hospital, Malmö, Sweden), Masoom Haider (University of Toronto, Toronto, Canada), Kees van Bochove (The Hyve B.V. Utrecht, Utrecht, the Netherlands), Ballentine Carter (Johns Hopkins University, Baltimore, USA).

Management team: Rachelle Kirk-Burnnand (Movember Foundation, Melbourne, Australia), Sam Gledhill (Movember
Foundation, Melbourne, Australia), Mark Buzza (Movember Foundation, Melbourne, Australia), Sophie Bruinsma (Erasmus Medical Center, Rotterdam, The Netherlands).

Correspondence: Sophie Bruinsma, Department of Urology, Erasmus MC, P.O. Box 2014, 3000 CA Rotterdam,

The Netherlands.

e-mail: s.m.bruinsma@erasmusmc.nl

Abbreviations: AS, active surveillance; GAP3, Global Action Plan Prostate Cancer Active Surveillance initiative; IQR, interquartile range; $\mathrm{PCa}$, prostate cancer.

\section{Supporting Information}

Additional Supporting Information may be found in the online version of this article:

Table S1. Participating centers and number of patients. Table S2. Protocol overview: Recommendations for patient selection for each participating center.

Table S3. Protocol overview: Recommendations for follow-up monitoring for each participating center.

Table S4. Characteristics at time of PCa diagnosis for all men included in the GAP3 cohort for each participating center. 